\title{
CORRIGENDA
}

\section{Seasonal time bombs: dominant temperate viruses affect Southern Ocean microbial dynamics}

Jennifer R Brum, Bonnie L Hurwitz, Oscar Schofield, Hugh W Ducklow and Matthew B Sullivan

The ISME Journal (2017) 11, 588; doi:10.1038/ismej.2016.126

Correction to: The ISME Journal (2016) 10, 400-415; doi:10.1038/ismej.2015.121; published online 21 August 2015

Updated online 21 September 2016: This article was originally published under NPG's License to
Publish, but has now been made available under a CC BY-NC-SA 4.0 license. The PDF and HTML versions of the paper have been modified accordingly.

\section{Global occurrence and heterogeneity of the Roseobacter-clade species Ruegeria mobilis}

Eva C Sonnenschein, Kristian F Nielsen, Paul D’Alvise, Cisse H Porsby, Jette Melchiorsen, Jens Heilmann, Panos G Kalatzis, Mario López-Pérez, Boyke Bunk, Cathrin Spröer, Mathias Middelboe and Lone Gram

The ISME Journal (2017) 11, 588; doi:10.1038/ismej.2016.125

Correction to: The ISME Journal (2017) 11, 569-583; doi:10.1038/ismej.2016.111; published online 23 August 2016

The affiliation listed for Boyke Bunk and Cathrin Spröer was incorrectly listed as 'Helmholtz Centre for Infection Research, Braunschweig, Germany’.
The correct affiliation is 'Leibniz-Institute DSMZDeutsche Sammlung von Mikroorganismen und Zellkulturen GmbH, Braunschweig, Germany’. 